05

\title{
Упрочнение поверхностного слоя металлических изделий воздействием плазмы комбинированного газового разряда
}

\author{
(C) Б.М. Бржозовский, ${ }^{1}$ С.Г. Гестрин, ${ }^{1,2}$ Е.П. Зинина, ${ }^{1}$ В.В. Мартынов ${ }^{1}$ \\ ${ }^{1}$ Саратовский государственный технический университет им. Гагарина Ю.А., \\ 410054 Саратов, Россия \\ ${ }^{2}$ Саратовский государственный аграрный университет им. Н.И. Вавилова, \\ 410012 Саратов, Россия \\ e-mail: gestrin.s@yandex.ru
}

(Поступило в Редакцию 7 марта 2017 г.)

Изучены свойства комбинированного газового разряда пониженного давления, возникающего в резонаторной камере вблизи поверхности обрабатываемого металлического изделия при наложении сверхвысокочастотного электромагнитного и электростатического полей. Показано, что при подаче на образец постоянного потенциала вблизи него формируется приповерхностный слой, в котором в зависимости от знака потенциала происходит ускорение положительных ионов или электронов до энергий, составляющих сотни $\mathrm{eV}$. Взаимодействие ускоренных частиц с образцом приводит к выделению в тонком приповерхностном слое количества теплоты, достаточного для его расплавления. В случае подачи положительного потенциала возникает упрочняющая поверхность изделия нанокомпозитная структура. Получены оценки физических параметров комбинированного газового разряда.

DOI: 10.21883/JTF.2017.12.45210.2240

\section{Введение}

Современный этап развития производства в различных отраслях характеризуется ужесточением условий эксплуатации изделий, особенно геометрически сложных, из металлических материалов и сплавов [1-5]. Изделия эксплуатируются в условиях действия повышенных температур, силовых нагрузок, агрессивных сред. В связи с этим актуальной является проблема повышения их эксплуатационной надежности по параметрам коррозионной и абразивной стойкости.

Перспективным направлением решения проблемы повышения эксплуатационной надежности является создание технологий и оборудования, которые позволяют упрочнять поверхностный слой изделий. Одной из таких технологий является воздействие на изделие плазмой комбинированного разряда пониженного давления $[1,2]$. Данное воздействие отличается от аналогов тем, что плазма генерируется непосредственно вокруг обрабатываемой поверхности при наложении стационарного электрического и СВЧ электромагнитного полей, что позволяет формировать в поверхностном слое нанокомпозитную структуру. Структура состоит из частиц диаметром $\sim 20-100 \mathrm{~nm}$ (кластеров), способных устойчиво противодействовать внешним силовым возмущениям, и частиц с размерами менее $10 \mathrm{~nm}$ (аморфной связки), образующих прослойку, способную перестраиваться в направлении действия внешних сил с постепенным уменьшением свободного объема в виде пустот между ее атомами. Кроме этого, в отличие от других плазменных технологий воздействие плазмы позволяет формировать градиентный переход (подслой) к материалу основы, вызывающий изменение ее химического состава и размеров зеренной структуры, что является фактором дополни- тельного повышения прочностных характеристик, в том числе адгезионных. В условиях эксплуатации изделия такая структура обеспечивает повышенную диссипацию энергии, образование упорядоченных, самоорганизующихся структур трения, что повышает устойчивость изделия к внешним воздействиям, влияющим на характеристики их прочности и твердости.

Ниже подробно описана экспериментальная установка, использованная для упрочнения поверхностного слоя металлических изделий (разд. 1), а также проанализированы свойства комбинированного газового разряда, возникающего в резонаторной камере установки (разд. 2-4). Показано, что при подаче на обрабатываемое изделие постоянного отрицательного потенциала $\varphi_{0}<0$, называемого в дальнейшем потенциалом смещения, возникающий комбинированный разряд в значительной степени обладает свойствами ВЧ разряда $(1-100 \mathrm{MHz})$, изученного ранее в работе [5]. Вблизи поверхности изделия формируется мощный приповерхностный слой положительного пространственного заряда, в котором происходит ускорение положительных ионов до высоких энергий $\varepsilon \sim 300 \mathrm{eV}$.

Если $\varphi_{0}=0$, то, как нами показано, между плазмой и поверхностью изделия возникает разность потенциалов $U_{p}=-\langle V\rangle$, где $\langle V\rangle<0-$ среднее по времени значение СВЧ составляющей потенциала образца. Значение $U_{p} \sim 15 \mathrm{~V}$ относительно невелико, что связано с малой толщиной приповерхностного слоя $d$, и существенно меньше, чем то, которое характерно для ВЧ разряда, где оно достигает сотен вольт [5].

Если $\varphi_{0}>0$ и выполняется условие $\varphi_{0}>|\langle V\rangle|$, то вблизи изделия формируется анодный слой, в котором электроны ускоряются до энергий $\varepsilon \approx 200 \mathrm{eV}$. 
В разд. 5 получены оценки характерных параметров газового разряда в случае нулевого потенциала смещения $\varphi_{0}$ на образце (подраздел 5.1), а также при подаче на образец отрицательного (подраздел 5.2) и положительного (подраздел 5.3) потенциалов смещения.

\section{1. Экспериментальная установка}

В состав установки входят: цилиндрический резонатор, внутренний вакуумируемый объем которого ограничен радиопрозрачным цилиндром; система возбуждения резонатора, включающая свернутую в кольцо волноводно-щелевую антенну; генератор СВЧ энергии с источником питания; блок подачи потенциала на обрабатываемый объект посредством держателя, расположенного в вакуумируемой части цилиндрического резонатора; вакуумную систему; систему напуска газа [6]. В конструкции установки отсутствует традиционный катодный узел как конструктивный элемент, эмитирующий электроны.

Источник микроволновой энергии состоит из блока питания и генератора СВЧ. Генератор СВЧ разработан на базе серийно-выпускаемого магнетрона типа M-105 с выходной мощностью до $700 \mathrm{~W}$ и частотой генерации в диапазоне от 2401 до $2499 \mathrm{MHz}$. Схема блока питания генератора СВЧ разработана на основе трансформатора-регулятора с магнитной коммутацией, позволяющего осуществлять плавную регулировку подводимой СВЧ мощности, обеспечивать ее высокую стабильность (в пределах 3\% при отклонениях напряжения питающей сети на $\pm 10 \%$ от номинального значения), а также управлять режимами работы от ЭВМ.

Напуск плазмообразующего газа осуществляется через систему трубопроводов. По показаниям вакуумметра ведется контроль давления в резонаторной камере, с помощью регулятора расхода газа задается уровень натекания газа. Подача постоянного потенциала смещения $\varphi_{0}$ на обрабатываемый инструмент осуществляется от источника питания постоянного тока типа Б5-50. Энергопотребление установки в целом не превышает $2 \mathrm{~kW}$.

Контроль выходных параметров осуществляется по току смещения с помощью вольтамперметра М-62 (класс точности 0.2) и лаговой температуре через систему автоматического контроля на базе модуля ввода сигналов термопар ADAM-4018. Помимо этого, в процессе плазменной обработки с помощью миниатюрной видеокамеры может проводиться видеонаблюдение с последующей регистрацией видеофайлов.

Более детально конструкция резонатора и рабочей камеры показана на рис. 1. Обрабатываемое изделие 1 закрепляется в сменной цанге 2 держателя 3, расположенного в рабочей камере 4. Часть поверхности обрабатываемого изделия и цанга 2 изолируются радиопрозрачным материалом 5; открытой остается только поверхность, подлежащая обработке. Рабочая камера откачивается с помощью вакуумной системы 6 до

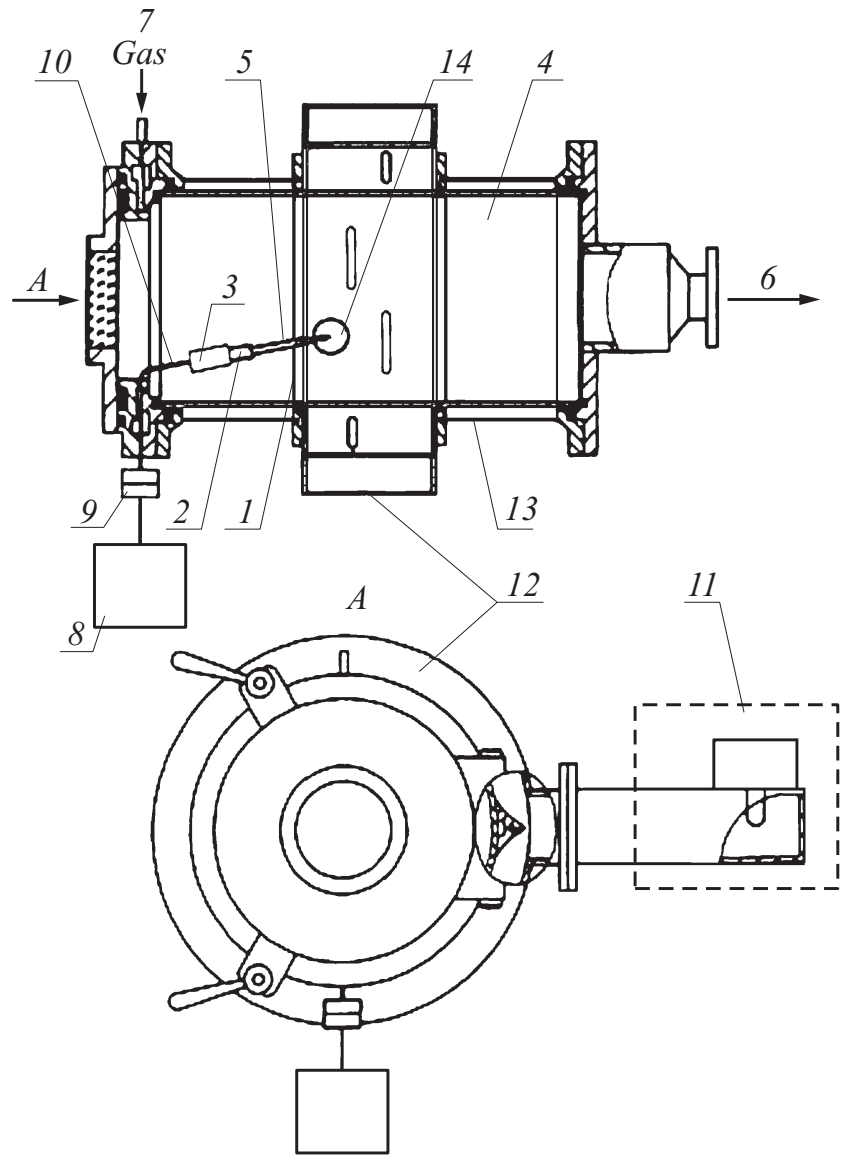

Рис. 1. Схематичное изображение продольного сечения резонатора.

предельного давления и через систему газонапуска 7 в ней устанавливается рабочее давление технологического газа (азот). На изделие подается положительный потенциал определенной величины от блока подачи потенциала 8 через герметичный разъем 9, кабель с многослойными элементами защиты 10 и держатель 3. От генератора СВЧ энергии 11 через волноводнощелевую антенну 12 , свернутую в кольцо, в цилиндрическом резонаторе 13 возбуждаются СВЧ электромагнитные колебания. При наложении электростатического и СВЧ электромагнитного полей вблизи открытой части обрабатываемого изделия возникает комбинированный газовый разряд пониженного давления 14. Минимальные значения, при которых происходит формирование плазмы вокруг изделия при рабочем давлении $300 \mathrm{~Pa}$ составляют: потенциал смещения $-50 \mathrm{~V}$, подводимая СВЧ-мощность - $20 \mathrm{~W}$.

Специально разработанный контактно-термопарный узел 3 выполняет следующие функции: измерение температуры на инструменте в процессе обработки; подача потенциала смещения на инструмент; защита от утечек СВЧ энергии.

Высокая эффективность энергетического вклада СВЧ энергии в разряд при работе на малых уровнях СВЧ 
мощности позволила упростить СВЧ тракт и не устанавливать дополнительных элементов защиты магнетрона от отраженной мощности, а также дополнительных подстроечных элементов для уменьшения потерь в резонаторе.

\section{2. Комбинированный газовый разряд в резонаторе}

Рассмотрим процессы, протекающие в плазменном облаке, сформированном при наложении электростатического поля и СВЧ электромагнитного поля в среде технологического газа, давление которого $p \sim 300 \mathrm{~Pa}$ непосредственно вокруг обрабатываемого изделия. Уровень СВЧ мощности составляет порядка $100 \mathrm{~W}$. Видимая область облака имеет характерный диаметр $L \sim 4 \mathrm{~cm}$ (рис. 2). По своим характеристикам комбинированный газовый разряд является СВЧ разрядом пониженного давления (обычно к таким разрядам относят разряды, возбуждаемые быстропеременным электрическим полем в диапазоне частот $v=10^{9}-10^{11} \mathrm{~Hz}$ с длинами волн $\lambda=0.3-30 \mathrm{~cm})$, причем существенное влияние на него, как будет показано ниже, оказывает наличие стационарного электрического поля.

Разряд возбуждается магнетроном и возникает во внутрирезонаторном пространстве. Источник возбуждения резонатора (магнетрон $\mathrm{c}$ рабочей частотой $v=2.45 \mathrm{GHz}$ и длиной волны $\lambda=12.5 \mathrm{~cm}$ ) определяет его рабочую моду. Размер обрабатываемого образца $d_{u} \sim 1 \mathrm{~cm}$ мал по сравнению с размерами резонатора $(D=15 \mathrm{~cm})$. При внесении образца в рабочую камеру СВЧ поле перестроится в некоторой прилегающей к нему области, в результате чего появятся линии напряженности, начало и конец которых будет приходиться

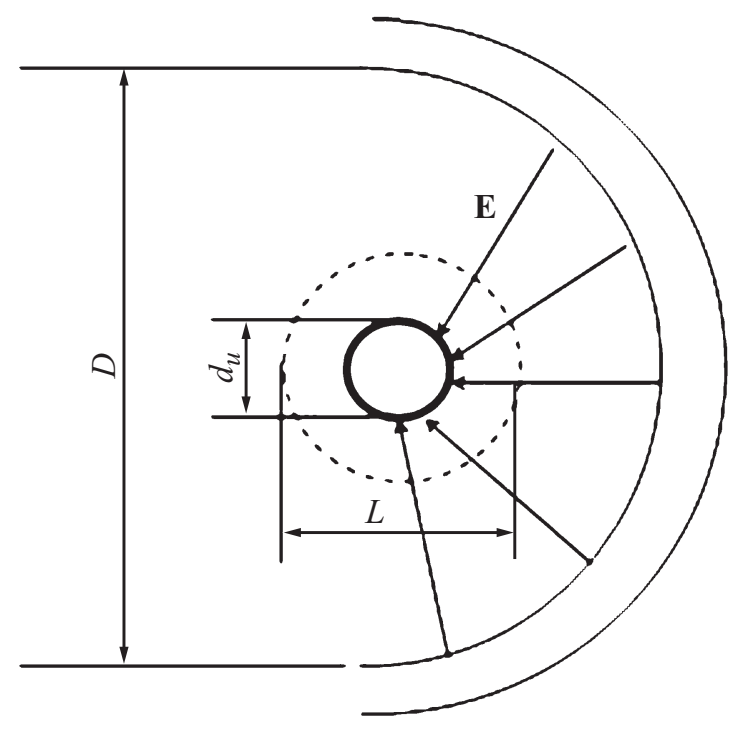

Рис. 2. Электрическое поле и облако плазмы вблизи образца, размещенного в резонаторной камере. на образец и близко к нему расположенный участок стенки рабочей камеры (рис. 2). Далее будет показано, что при определенных условиях в этом случае будет возникать диодный эффект, аналогичный тому, который наблюдается при ВЧ разряде [5,7].

Диодный эффект проявляется в образовании пристеночного слоя положительного пространственного заряда c большой постоянной разностью потенциалов между плазмой и стенкой $U p \gg k_{B} T_{e}\left(k_{B}-\right.$ постоянная Больцмана, $T_{e}, e-$ температура и заряд электронов). Величина $U_{p}$ зависит от амплитуды напряженности $E_{0}$ рабочей моды СВЧ электрического поля, а также от величины отрицательного потенциала $\varphi_{0}$.

\section{3. Приповерхностный слой положительного пространственного заряда}

Наличие приповерхностных слоев приводит к тому, что пространственное распределение потенциала плазмы принимает форму потенциальной ямы, в которой находятся электроны. Потенциальный барьер на краю ямы совершает СВЧ колебания относительно своего среднего значения, которое равно $U_{p}=-\langle V\rangle-\varphi_{0}$. В моменты исчезновения барьера электроны получают кратковременный доступ к поверхности образца. Возникающий при этом ток электронов компенсирует постоянный в среднем по времени ток ионов.

Положительный ион передает свой заряд образцу, присоединяя к себе один из его электронов проводимости. Находясь еще на некотором расстоянии от его поверхности, ион создает электрическое поле, вытягивающее электроны. Процесс этот происходит без изменения полной энергии электрона за счет туннельного перехода в область вблизи иона. Электрон, покидающий металл, захватывается положительным ионом. Здесь электрон занимает какой-либо из квантовых уровней энергии. При этом возникает нейтральная молекула в нормальном или возбужденном состоянии.

Объем квазинейтральной плазмы комбинированного разряда, для которого характерен амбиполярный режим диффузии заряженных частиц, отделен от образца переходным слоем, в котором нарушается квазинейтральность и сосредоточено основное падение потенциала постоянного поля.

Зондовые исследования приповерхностных профилей потенциала и плотности плазмы в диодном слое показывают, что такие параметры слоя как плотность ионного тока через слой $j_{i}$, его ширина $d$ и потенциал $U_{p}$ связаны соотношением, похожим на закон „трех вторых“ Чайльда-Ленгмюра, который справедлив для слоя положительного пространственного заряда в режиме насыщения тока ионов [5,7]. Сходство этих соотношений объясняется одинаковыми условиями ускорения ионов в постоянном и квазистационарном электрических полях. 


\section{4. Ускорение ионов в приповерхностном слое комбинированного разряда и аналог закона Чайльда-Ленгмюра}

В приповерхностном слое комбинированного разряда (рис. 3) положительные ионы захватываются и ускоряются падением стационарного потенциала плазмы $U_{p}=-\langle V\rangle-\varphi_{0}$. Концентрация ионов в слое $n_{i}$ становится неоднородной и резко спадает в направлении к поверхности образца. Энергия $\varepsilon_{i}$ ускоренных ионов зависит от давления газа и достигает величины $e U_{p}$, составляющей сотни $\mathrm{eV}$.

Постоянный ионный ток $i$ из слоя на поверхность образца, находящуюся в среднем под большим отрицательным потенциалом относительно квазинейтральной плазмы, в отсутствие ионизации в слое не зависит от величины потенциала $U_{p}$.

Ионный ток на поверхность образца определяется только диффузионным потоком ионов из квазинейтральной плазмы, равным ионному току насыщения ленгмюровского зонда. Распределение постоянного потенциала и профиль ионной концентрации в слое формируются аналогично ленгмюровскому слою в режиме ионного тока насыщения (рис. 3). Отличие заключается лишь в том, что объемный заряд ионов в СВЧ приповерхностном слое частично компенсируется электронами плазмы, попадающими в процессе колебаний в слой, что приводит к тому, что ширина СВЧ приповерхностного слоя при прочих равных условиях оказывается больше ширины ленгмюровского слоя [5]. Экспериментальные исследования и теоретические расчеты [5,7] показывают, что стационарные параметры приповерхностного слоя комбинированного разряда $U_{p}$ и $d$ связаны между собой соотношением, родственным закону Чайльда-Ленгмюра.

В диапазоне давлений газа $p=10^{-2}-10 \mathrm{~mm} \mathrm{Hg}$ приповерхностный слой не является бесстолкновительным. Отношение длины свободного пробега ионов $\lambda_{i}$ к $d$ при этих давлениях составляет $1-10^{-2}$. В работе [7] получена приближенная формула для СВЧ аналога закона Чайльда-Ленгмюра:

$$
U_{p}=\frac{6}{5}\left(\frac{m_{i}}{2 e}\right)^{1 / 3}\left(\frac{3}{2} \frac{j_{i}}{\varepsilon_{0}}\right)^{2 / 3}\left(\frac{d+\lambda_{i}}{2 d+\lambda_{i}}\right)\left(\frac{d+\lambda_{i}}{\lambda_{i}}\right)^{1 / 3} d^{4 / 3},
$$

где $m_{i}$ - масса иона, $\varepsilon_{0}=8.85 \cdot 10^{-12} \mathrm{~F} / \mathrm{m}$ - электрическая постоянная.

При комбинированном разряде происходит мощная ионная бомбардировка поверхности образца, под действием которой может развиваться вторичная ионноэлектронная эмиссия, существенно влияющая на механизм разряда (рис. 3). Основное количество эмиссионных $\gamma$-электронов поступает с поверхности образца, которая подвергается наиболее интенсивной бомбардировке. Эти электроны могут ускоряться в приповерхностном падении потенциала до энергий порядка $e U_{p}$,

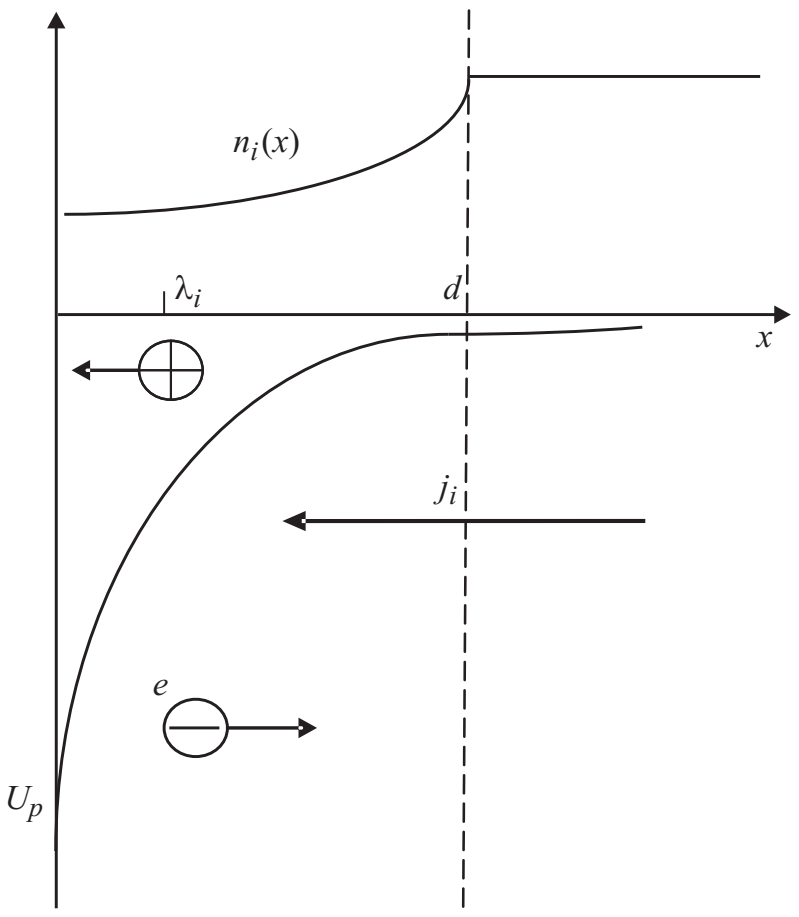

Рис. 3. Распределение постоянного потенциала $U_{p}$ и профиль ионной концентрации $n_{i}(x)$ в приповерхностном слое.

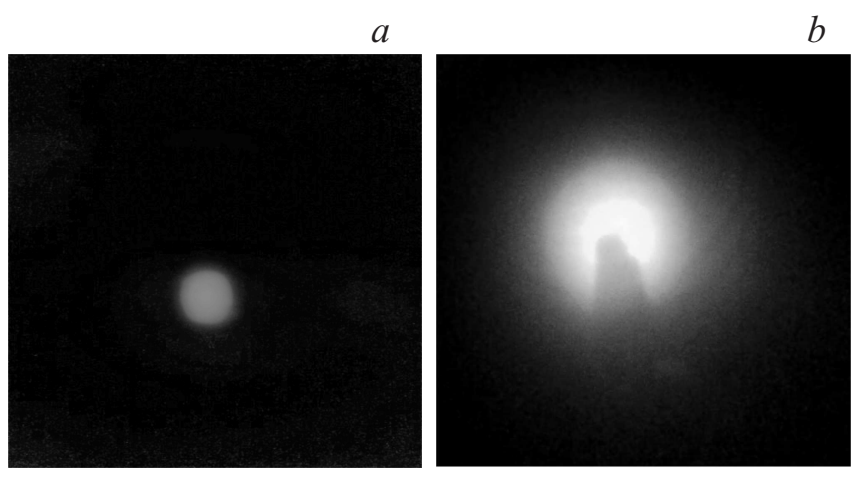

Рис. 4. Плазменный разряд, полученный без образца (a) и при его помещении в рабочую камеру и подаче на него отрицательного потенциала смещения $\varphi_{0}<0(b)$.

составляющих несколько сотен $\mathrm{eV}$. Данная энергия значительно превосходит энергию объемных $\alpha$-электронов, совершающих колебания в СВЧ электрическом поле на дне потенциальной ямы и обладающих низкой тепловой энергией $(1-5 \mathrm{eV})$.

В зависимости от давления газа и длины свободного пробега электроны преимущественно рассеиваются либо в глубине разряда, либо в приповерхностном слое, что определяет режим горения СВЧ разряда и его пространственную структуру. Принято различать сильноточный $\gamma$ - и слаботочный $\alpha$-разряды. Экспериментально обнаружено, что при $p>1 \mathrm{~mm} \mathrm{Hg}$ и напряжении $U_{p} \sim 300-400 \mathrm{~V}[5]$ наблюдается резкое повышение 
разрядного тока и переход разряда в сильноточный $\gamma$ режим, что связано с изменением характера ионизационных процессов в приповерхностном слое.

Проведенные эксперименты показали, что в отсутствие обрабатываемого образца в пространстве рабочей камеры возникает слабый СВЧ разряд оранжевого цвета, что свидетельствует о наличии плазмы низкой концентрации (рис. $4, a)$.

При сильноточном $\gamma$-разряде свечение локализуется вблизи поверхности образца и состоит из нескольких слоев различного свечения (рис. $4, b$ ).

\section{5. Оценки параметров комбинированного газового разряда}

Основываясь на соотношении (1), приведем оценки параметров комбинированного газового разряда.

5.1. Рассмотрим вначале случай, когда потенциал смещения $\varphi_{0}=0$. После подачи напряжения питания на магнетрон очень быстро (за время $\sim 10^{-8}-10^{-7} \mathrm{~s}$ ) происходит установление частоты колебаний магнетрона, соответствующей резонансной частоте системы, образованной магнетроном, объемным СВЧ резонатором и образцом, размещенным внутри резонатора. После этого начинается накопление энергии внутри объемного резонатора. Напряженность поля внутри резонатора при этом возрастает до тех пор, пока не превысит пробойное значение для заполняющего его внутреннее пространство воздуха. После этого внутри резонатора вблизи образца зажигается разряд. В отсутствие электростатического поля $\left(\varphi_{0}=0\right)$ комбинированный разряд является в чистом виде СВЧ газовым разрядом.

Для оценки значения $E_{0}$ воспользуемся соотношением

$$
W=\frac{\varepsilon_{0} E_{0}^{2}}{2} V=\frac{P Q_{0}}{\omega},
$$

откуда

$$
E_{0}=\sqrt{\frac{2 P Q_{0}}{\varepsilon_{0} V \omega}} .
$$

Здесь $W$ - запасенная в резонаторе электромагнитная энергия, $Q_{0}$ - добротность резонатора, $V$ - объем резонатора, $P$ - мощность, отдаваемая магнетроном, $\omega$ - циклическая частота. Из (3) для значений параметров $P=100 \mathrm{~W}, V=5 \cdot 10^{-3} \mathrm{~m}, Q_{0}=10^{5}$, $\omega=1.51 \cdot 10^{10} \mathrm{~s}^{-1}$ находим $E_{0}=1.7 \cdot 10^{5} \mathrm{~V} / \mathrm{m}$, что превосходит порог СВЧ пробоя воздуха, который в зависимости от давления, частоты и разрядного объема может достигать $\sim 10^{5} \mathrm{~V} / \mathrm{m}$.

Для газа пробой означает переход в ионизированное состояние. При этом возрастание тока приводит к еще большему возрастанию концентрации ионов и проводимости газа, а следовательно и к понижению напряжения, которое требуется для поддержания такого тока. После зажигания СВЧ разряда энергия, запасенная в резонаторе, уменьшается, а вместе с ней уменьшается и напряженность электрического поля $E_{0}$, а также определяемое ею значение СВЧ потенциала на поверхности образца $U_{0}$ до значений порядка несколько сотен вольт.

Наличие СВЧ электрического поля в резонаторе ответственно не только за поддержание плазмы и СВЧ разряда в рабочей камере, но и за образование пристеночного слоя. На частотах поля порядка $v=10^{9}-10^{11} \mathrm{~Hz} \mathrm{CBЧ}$ ток в плазме протекает за счет колебаний электронов. Смещение электрона в высокочастотном поле

$$
x=\frac{e}{m_{e} \omega^{2}} E_{0} \exp (-i \omega t),
$$

где $m_{e}-$ масса электрона, $\omega=2 \pi \nu$. Смещением тяжелых ионов, которое $\sim e / m_{i} \omega^{2}$, можно пренебречь, так как $m_{e} \ll m_{i}$. За период поля ионы можно считать покоящимися. Пристеночный слой плазмы, прилегающий к обрабатываемому образцу, имеет ширину порядка амплитуды пространственных колебаний электронов $d \sim e E_{0} / m_{e} \omega^{2}$. Для значений $e=1.6 \cdot 10^{-19} \mathrm{C}, m_{e}=9.1 \cdot 10^{-31} \mathrm{~kg}, \omega=1.51 \cdot 10^{10} \mathrm{~Hz}$, $E_{0}=3.9 \cdot 10^{5} \mathrm{~V} / \mathrm{m}$ находим $d \sim 0.3 \cdot 10^{-3} \mathrm{~m}$.

Концентрация нейтральных молекул $n=p / k_{B} T$. Для $p=0.00263 \cdot 10^{5} \mathrm{~Pa}(2 \mathrm{~mm} \mathrm{Hg})$ и $T=500 \mathrm{~K}$ - температура нейтральных молекул, находим $n \sim 3.8 \cdot 10^{22} \mathrm{~m}^{-3}$.

Скорость теплового движения электронов $\left\langle v_{e}\right\rangle=$ $=\sqrt{3 k_{B} T_{e} / m_{e}}$ при температуре электронов $T_{e}=2 \cdot 10^{4} \mathrm{~K}$ достигает значения $\left\langle v_{e}\right\rangle=0.94 \cdot 10^{6} \mathrm{~m} / \mathrm{s}$. Энергия теплового движения электрона в объеме квазинейтральной плазмы $\varepsilon=3 k_{B} T_{e} / m_{e} \sim 2.6 \mathrm{eV}$.

Для оценки значения ионного тока насыщения $j_{i}$ можно использовать полуэмпирическую формулу Бома

$$
j_{i}=e n_{i}(d) v_{i 0}=e n_{0 i} e^{-1 / 2} v_{i 0}=0.6 e n_{0 i} \sqrt{\frac{k_{B} T_{e}}{m_{i}}} .
$$

Здесь $n_{i}(d)$ - концентрация ионов на границе приповерхностного слоя, $n_{0 i}$ - концентрация ионов на расстояниях $x \gg d$ от образца, $v_{0 i}=\sqrt{k_{B} T_{e} / m_{i}}-$ скорость ионов на границе слоя, определяемая критерием Бома. Для $m_{i}=2.325 \cdot 10^{-26} \mathrm{~kg}$ - масса иона азота - и $T_{e}=2 \cdot 10^{4} \mathrm{~K}$ скорость ионов $v_{i 0}=3.45 \cdot 10^{3} \mathrm{~m} / \mathrm{s}$.

Процессы, определяемые параметрами, одни из которых характерны для электронов (в нашем случае $T_{e}$ ), а другие - для ионов (в формуле $(5)$ - масса иона $\left.m_{i}\right)$, обычно называются амбиполярными.

При силе тока, протекающего через установку, $i \sim 12 \mathrm{~mA}$ и площади поверхности образца $S=$ $=6 \cdot 10^{-4} \mathrm{~m}^{2}$ плотность тока составляет $j_{i}=20 \mathrm{~A} / \mathrm{m}^{2}$.

Из (5) находим концентрацию ионов $n_{0 i}=6 \cdot 10^{16} \mathrm{~m}^{3}$. Плазменная частота для электронов $\omega_{p}=\sqrt{n_{e} e^{2} / \varepsilon_{0} m_{e}}=$ $=1.38 \cdot 10^{10} \mathrm{~Hz}$. Частота магнетрона $\omega=2 \pi c / \lambda=$ $=1.51 \cdot 10^{10} \mathrm{~Hz}$. Таким образом, СВЧ поле может проникать через плазму, так как $\omega>\omega_{p}$.

Длина свободного пробега иона $\lambda=1 / \sqrt{2} n \sigma=$ $=6.6 \cdot 10^{-5} \mathrm{~m}$, где $\sigma=\pi d_{0}^{2}-$ площадь эффективного поперечного сечения соударения молекул азота, $d_{0}=3 \cdot 10^{-10} \mathrm{~m}$ - эффективный диаметр молекул азота. 
Для $\lambda_{i}=6.6 \cdot 10^{-5} \mathrm{~m}, m_{i}=2.325 \cdot 10^{-26} \mathrm{~kg}, d \sim 0.3$ $\times 10^{-3} \mathrm{~m}$ из (1) получим $U_{p} \sim 16.82 \mathrm{~V}$. При этом $\lambda_{i} / d \sim 0.22$. Заметим, что полученное значение $U_{p}$ относительно невелико, что связано с малой толщиной приповерхностного слоя $d$, и существенно меньше, чем то, которое характерно для ВЧ разряда, где оно достигает сотен вольт.

5.2. Если на образец подать постоянный отрицательный потенциал $\varphi_{0}$, то ситуация существенно меняется (рис. 5,a). Потенциал образца $\varphi(t)=V(t)+\varphi_{0}$ в этом случае представляет собой сумму СВЧ составляющей $V(t)$, среднее по времени значение которой $\langle V\rangle$, и постоянного потенциала $\varphi_{0},\langle V\rangle+\varphi_{0}=-U_{p}$.

На рис. 5, a приведены также зависимости СВЧ потенциала $U(t)$, максимальное значение которого $U_{0}$ определяется амплитудой напряженности $E_{0}$ рабочей моды СВЧ электрического поля в резонаторе и ширины приповерхностного слоя $d(t)$.

Пусть $\varphi_{0}=-300 \mathrm{~V}$. Из (1) для стационарного потенциала $U_{p}=-\langle V\rangle-\varphi_{0}=300+16.82=-316.82 \mathrm{~V}$ находим толщину слоя $d=1.9 \cdot 10^{-3} \mathrm{~m}$.

Число ионов, поступающих в единицу времени на поверхность образца: $d N_{i} / d t=j_{i} S / e=5 \cdot 10^{16} \mathrm{~s}^{-1}$.

Энергия направленного движения, приобретенная ионом в приповерхностном слое, $\varepsilon_{i} \approx e \varphi=316.82 \mathrm{eV} \approx$ $\approx 5 \cdot 10^{-17} \mathrm{~J}$. Скорость направленного движения иона в конце разгона $v_{i} \approx \sqrt{2 e \varphi / m_{i}}=6.5 \cdot 10^{4} \mathrm{~m} / \mathrm{s}$.

Если предположить, что вся энергия $\varphi_{i}$ передается от иона к образцу, то в единицу времени на поверхность образца попадет $d W_{i} / d t=2.5 \mathrm{~W}$.

Энергия, поступающая на поверхность образца за характерное время обработки $\tau=1000 \mathrm{~s}, W \sim 2500 \mathrm{~J}$. Таким образом, за время обработки в образце выделяется количество теплоты $Q \sim 2500 \mathrm{~J}$.

Потенциальный барьер вблизи образца совершает СВЧ колебания относительно своего среднего значения, которое равно $U_{p}$. В моменты исчезновения барьера осуществляется кратковременный импульсный сток электронов на поверхность образца, компенсирующий постоянный в среднем по времени ток ионов.

5.3. Рассмотрим ситуацию, возникающую при подаче на образец положительного потенциала $\varphi_{0}$. Если $\varphi_{0}>\langle V\rangle$, то средний потенциал квазинейтральной плазмы относительно образца $U_{p}<0$. Зависимости потенциала на поверхности образца и толщины приповерхностного слоя от времени при подаче на образец положительного потенциала изображены на рис. $5, b$.

Средняя скорость теплового движения электронов, как показано выше, составляет $\left\langle v_{e}\right\rangle \sim 0.94 \cdot 10^{6} \mathrm{~m} / \mathrm{s}$. Ток, протекающий через установку:

$$
i=j S=\frac{1}{4} n_{e} e\left\langle v_{e}\right\rangle S,
$$

где $S$ - площадь поверхности обрабатываемого образца. Для тока $i \sim 8 \mathrm{~mA}$ находим значение концентрации электронов: $n_{e}=3.54 \cdot 10^{14} \mathrm{~m}^{-3}$.
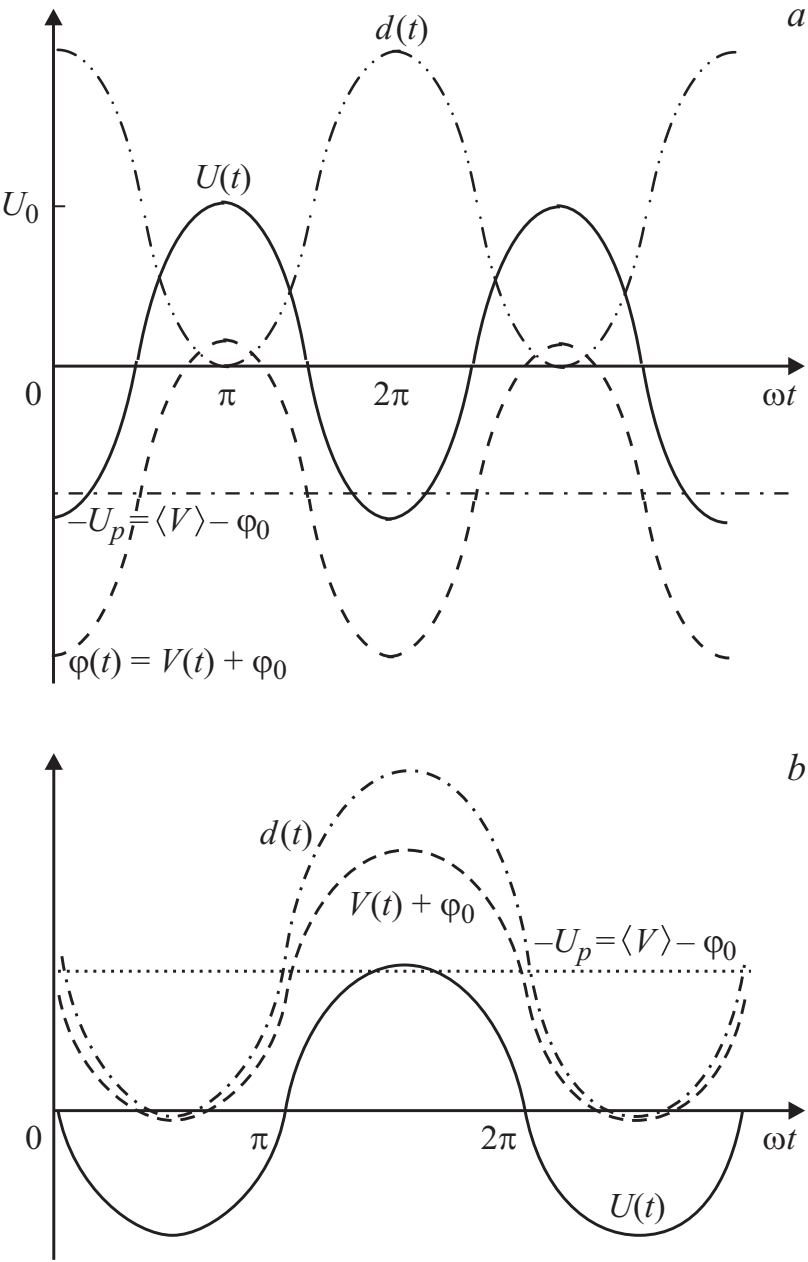

Рис. 5. Зависимости от времени толщины приповерхностного слоя $d(t)$, СВЧ потенциала электрического поля $\left.U_{(} t\right)$ и потенциала образца $\varphi(t)$ при подаче на образец потенциала смещения $\varphi_{0}<0(a)$ и $\varphi_{0}>0(b)$.

Число электронов, поступающих в единицу времени на поверхность изделия, $d N_{i} / d t=5 \cdot 10^{16} \mathrm{~s}^{-1}$.

Энергия направленного движения электрона, которую он приобретает в предслое

$$
k_{B} T_{i}=m_{e} v_{0 e}^{2},
$$

Отсюда для $T_{i}=500 \mathrm{~K}$ находим $v_{0 e}=\sqrt{k_{B} T_{i} / m_{e}}=$ $=0.87 \cdot 10^{5} \mathrm{~m} / \mathrm{s}$. Таким образом, начальная скорость упорядоченного движения по направлению к аноду, которую имеет электрон при подлете из предслоя к оболочке, $v_{0 e} \approx 0.1\left\langle v_{e}\right\rangle$.

Энергия направленного движения, приобретенная электроном в электрическом поле вблизи поверхности образца, при $U_{p}=200 \mathrm{~V}$ составляет $\varepsilon_{e} \approx e U_{p}=$ $=200 \mathrm{eV}=3.2 \cdot 10^{-17} \mathrm{~J}$.

Таким образом, электроны набирают энергию и вызывают наблюдаемое в эксперименте анодное свечение малинового цвета (рис. 6, a). 

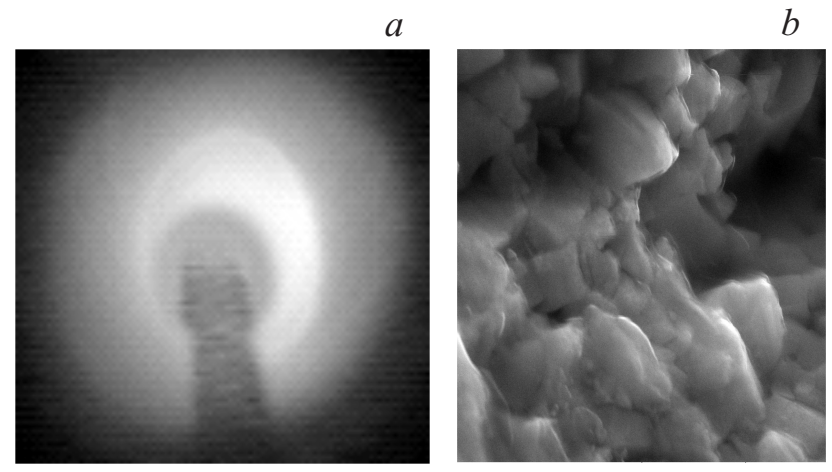

Рис. 6. Плазменный разряд в случае подачи на образец положительного потенциала смещения $\varphi_{0}>0(a)$ и застывший расплав на поверхности образца $(b)$.

Скорость направленного движения, приобретенная электроном после прохождения слоя, в 10 раз превосходит скорость теплового движения $v_{2} \approx \sqrt{2 \varepsilon_{e} / m_{e}}=$ $=0.83 \cdot 10^{7} \mathrm{~m} / \mathrm{s} \approx 10\left\langle v_{e}\right\rangle$. Энергия, поступающая на поверхность образца за время $\tau=1000 \mathrm{~s}, W \sim 1600 \mathrm{~J}$. Таким образом, за время проведения эксперимента в образце выделяется количество теплоты $Q \sim 1600 \mathrm{~J}$.

Длина свободного пробега электрона составляет: $\lambda_{e}=1 / \sqrt{2} n \sigma=2.64 \cdot 10^{-4} \mathrm{~m}, \quad$ где $\sigma=\pi d_{0}^{2} / 4, \quad d_{0}=3$ $\times 10^{-10} \mathrm{~m}-$ эффективный диаметр молекул азота.

Воспользовавшись формулой (1), определим по порядку величины толщину слоя $d \sim 1 \mathrm{~cm}$, при этом отношение $\lambda_{e} / d \sim 0.02$, что соответствует области применимости (1).

Оценим количество теплоты, необходимое для расплавления тонкого поверхностного слоя образца с зафиксированной на шлифах толщиной $l=0.04 \mathrm{~mm}$ при значениях параметров, характерных для стали: удельная теплоемкость $c=462 \mathrm{~J} /(\mathrm{kg} \cdot \mathrm{K})$, удельная теплота плавления $r=82000 \mathrm{~J} / \mathrm{kg}$, плотность $\rho=7700 \mathrm{~kg} / \mathrm{m}^{3}$, $t_{\text {пл. }} \sim 1500^{\circ} \mathrm{C}$, минимальное значение, равное $50^{\circ} \mathrm{C}$, температура имеет на противоположном конце образца, закрепленного в держателе, где она измеряется при помощи термопары, следовательно, $\Delta T=1450^{\circ} \mathrm{C}$.

Тогда $Q=(c \Delta T+r) \rho S l \approx 140 \mathrm{~J}$, что на порядок меньше чем количество теплоты, выделяющееся в образце за время проведения эксперимента. На (рис. $6, b$ ) изображен застывший расплав на поверхности образца из твердого сплава.

\section{6. Электрический заряд образца}

Проанализируем изменение заряда на поверхности образца с течением времени. Для простоты предположим, что обрабатываемый образец имеет форму шарика. Его поверхность бомбардируется положительными ионами или электронами, ускоренными в приповерхностном слое и создающими ток $j_{i, e} S$ :

$$
j_{i, e} S=\sigma_{0 i, e} \frac{1}{4 \pi \varepsilon_{0}} \frac{q}{r_{b}^{2}} 4 \pi r_{b}^{2}=\sigma_{0 i, e} \frac{q}{\varepsilon_{0}} .
$$

Скорость изменения среднего заряда на образце

$$
\begin{aligned}
\frac{d q}{d t} & =-j_{i} S+i=-\sigma_{0 i} E 4 \pi r_{b}^{2}+\frac{\varphi_{0}-\varphi}{R} \\
& =-\sigma_{0 i} \frac{1}{4 \pi \varepsilon_{0}} \frac{q}{r_{b}^{2}} 4 \pi r_{b}^{2}+\frac{\varphi_{0}}{R}-\frac{1}{4 \pi \varepsilon_{0}} \frac{q}{r_{b} R} .
\end{aligned}
$$

Здесь $q-$ заряд шарика, $i$ - ток, протекающий в цепи „плазма-образец-земля“, имеющей сопротивление $R$, $\varphi$ - потенциал образца, $\varphi_{0}-$ потенциал на клемме источника питания, создающего потенциал смещения на образце, $\sigma_{0 i}-$ проводимость плазмы (ионная или электронная), $r_{b}$ - радиус шарика.

Уравнение (9) можно записать в виде

$$
\frac{d q}{d t}=-\frac{1}{\tau}+\frac{\varphi_{0}+\langle V\rangle}{R},
$$

где

$$
\tau=\frac{1}{\frac{\sigma_{0 i}}{\varepsilon_{0}}+\frac{1}{4 \pi \varepsilon_{0} r_{b} R}} .
$$

Полагая, что в начальный момент времени заряд на поверхности шарика $q(0)=0$, находим, что при $t \gg \tau$ заряд стремится к постоянному значению

$$
q_{\infty}=\frac{\left(\varphi_{0}+\langle V\rangle\right)}{R} \tau_{0}=\frac{4 \pi \varepsilon_{0} r_{b}}{1+4 \pi r_{b} R \sigma_{0 i}}\left(\varphi_{0}+\langle V\rangle\right) .
$$

Из (12) видно, что знак заряда $q_{\infty}$ определяется знаком $\left(\varphi_{0}+\langle V\rangle\right)$, а величина зависит от сопротивления $R$ и проводимости $\sigma_{0 i}$.

\section{Заключение}

Таким образом, в работе изучены свойства и произведены оценки физических параметров комбинированного газового разряда, воздействующего на поверхность образца.

Показано, что при подаче на образец достаточно большого отрицательного потенциала смещения вблизи его поверхности формируется слой положительного пространственного заряда, в котором происходит ускорение положительных ионов до энергий, составляющих несколько сотен $\mathrm{eV}$. В этом случае свойства комбинированного разряда в значительной степени сходны со свойствами ВЧ разряда. Если на изделие подан положительный потенциал, то происходит формирование анодного слоя, в котором электроны ускоряются до энергий порядка сотен $\mathrm{eV}$.

В работе получены оценки характерных параметров комбинированного газового разряда в случае отрицательного, нулевого и положительного потенциалов смещения на образце. 
Показано, что взаимодействие ускоренных частиц с образцом приводит к выделению в нем значительного количества теплоты, достаточного для расплавления поверхностного слоя, а в случае положительного потенциала смещения также к формированию нанокомпозитной упрочняющей структуры.

Работа подготовлена при поддержке гранта Российского научного фонда (проект № 15-19-00030).

\section{Список литературы}

[1] Brzhozovskii B., Martynov V., Zinina E., Brovkova M. // IOP Conf. Series: Materials Science and Engineering [Electronic resource]. 2016. Vol. 116. P. 012007. 7 p.

[2] Brzhozovsky B., Martynov V., Zinina E., Brovkova M., Bochkarev P. // IOP Conf. Series: Materials Science and Engineering [Electronic resource]. 2016. Vol. 140. P. 012002. $10 \mathrm{p}$.

[3] Абдуллин И.Ш., Хабатхузин А.А., Христолюбова В.И. // Упрочняющие технологии и покрытия. 2015. № 12 (132). C. 13-18.

[4] Lisovskiy V.A., Booth J.-P. // Phys. Surf. Engin. 2003. Vol. 1. $\mathrm{N}$ 1. P. 34-36.

[5] Фареник В.И. // ФІП ФИП РSЕ. 2004. Т. 2. № 1. С. $117-$ 145.

[6] Бржсозовский Б.М., Мартынов В.В., Зинина Е.П. Упрочнение режущего инструмента воздействием низкотемпературной плазмы комбинированного разряда. Саратов: Сарат. гос. техн. ун-т, 2009. 176 с.

[7] Будянский А.М. // II Всес. совещ. „Высокочастотный разряд“: тез. докл. Куйбышев, 1989. С. 15. 